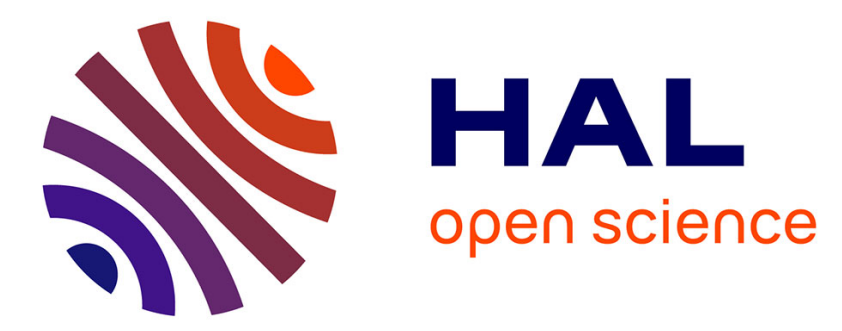

\title{
Chimiothérapie à hautes doses comme stratégie pour contourner la résistance aux médicaments dans les tumeurs solides
}

Frédéric Selle, Joseph Gligorov, Daniele G. Soares, Jean-Pierre Lotz

\section{- To cite this version:}

Frédéric Selle, Joseph Gligorov, Daniele G. Soares, Jean-Pierre Lotz. Chimiothérapie à hautes doses comme stratégie pour contourner la résistance aux médicaments dans les tumeurs solides. Bulletin du Cancer, 2016, 103 (10), pp.861-868. 10.1016/j.bulcan.2016.08.002 . hal-01380154

\section{HAL Id: hal-01380154 https://hal.sorbonne-universite.fr/hal-01380154}

Submitted on 12 Oct 2016

HAL is a multi-disciplinary open access archive for the deposit and dissemination of scientific research documents, whether they are published or not. The documents may come from teaching and research institutions in France or abroad, or from public or private research centers.
L'archive ouverte pluridisciplinaire HAL, est destinée au dépôt et à la diffusion de documents scientifiques de niveau recherche, publiés ou non, émanant des établissements d'enseignement et de recherche français ou étrangers, des laboratoires publics ou privés. 
CHIMIOTHERAPIE A HAUTES-DOSES COMME STRATEGIE POUR CONTOURNER LA RESISTANCE AUX MEDICAMENTS DANS LES TUMEURS SOLIDES

Titre court : Chimiothérapie à hautes-doses dans les tumeurs solides

HIGH-DOSE CHEMOTHERAPY AS A STRATEGY TO OVERCOME DRUG RESISTANCE IN SOLID TUMORS

Short title: High-doses chemotherapy in solid tumors

Frédéric SELLE ${ }^{1}$, Joseph GLIGOROV ${ }^{1,2}$, Daniele G. SOARES ${ }^{1}$, Jean-Pierre LOTZ ${ }^{1,2}$

1. Hôpital Tenon, Assistance Publique-Hôpitaux de Paris (AP-HP), Service d'Oncologie Médicale et de Thérapie Cellulaire; APREC (Alliance Pour la Recherche En Cancérologie),

2. Institut Universitaire de Cancérologie; Université Pierre et Marie Curie (UPMC Univ Paris 06); Sorbonne Universités, France

Auteur correspondant : Pr Jean-Pierre Lotz, Hôpital Tenon, Assistance Publique-Hôpitaux de Paris (AP-HP), Service d'Oncologie Médicale et de Thérapie Cellulaire; APREC (Alliance Pour la Recherche En Cancérologie), 4, rue de la Chine, 75970 Paris Cedex 20, France.

Tel: +33156016058

Fax: +33156016875

E-mail: jean-pierre.lotz@aphp.fr 
CHIMIOTHERAPIE A HAUTES-DOSES COMME STRATEGIE POUR CONTOURNER LA RESISTANCE AUX

MEDICAMENTS DANS LES TUMEURS SOLIDES

Titre court : Chimiothérapie à hautes-doses dans les tumeurs solides

HIGH-DOSE CHEMOTHERAPY AS A STRATEGY TO OVERCOME DRUG RESISTANCE IN SOLID TUMORS

Short title: High-doses chemotherapy in solid tumors

\begin{abstract}
Résumé
La chimiothérapie à hautes-doses a été développée dans les années 1980 basée sur des observations scientifiques faites in vitro. Il a été montré que la survie des cellules tumorales traitées par des agents alkylants respectait une forte relation dose-réponse. En plus, lorsque les doses étaient intensifiées, la résistance acquise des cellules tumorales pouvait être surmontée. En clinique, l'intensification de la dose des agents alkylants a permis d'accroitre les réponses thérapeutiques, au prix néanmoins d'une importante toxicité hématologique. Suite à l'avènement de l'autogreffe de cellules souches hématopoiétiques, les effets toxiques marqués associés à l'intensification thérapeutique ont été, dans leur majorité, contournés. En conséquence, les hautes-doses de chimiothérapie ont été évaluées dans différents types de tumeurs solides, en particulier dans les cancers du sein, de l'ovaire et dans les tumeurs germinales, mais aussi dans le cancer bronchique à petites cellules, les sarcomes des tissus mous et le sarcome d'Ewing. A ce jour, les hautes-doses de chimiothérapie avec le support des cellules souches hématopoïétiques ne sont utilisées que dans le cadre d'une thérapie de rattrapage pour les tumeurs germinales chimio-sensibles de mauvais pronostique. Pour ce qui concerne le traitement du cancer du sein et de l'ovaire, la chimiothérapie à hautes-doses ne doit être, aujourd'hui, envisagée que dans le cadre d'essais cliniques. Néanmoins, cette approche de thérapie intensive pour surmonter la résistance aux traitements standards reste toujours d'actualité. De nombreux efforts se poursuivent pour identifier de nouvelles combinaisons thérapeutiques actives à hautes-doses et capables d'améliorer les réponses.
\end{abstract}

Mots-clés: Hautes-doses de chimiothérapie; Tumeurs germinales; Autogreffe de cellules souches; Tumeurs solides; Cancer du sein; Cancer de l'ovaire 


\begin{abstract}
The concept of high-doses chemotherapy was developed in the 1980s based on in vitro scientific observations. Exposure of tumor cells to increasing concentrations of alkylating agents resulted in increased cell death in a strong dose-response manner. Moreover, the acquired resistance of tumor cells could be overcome by dose intensification. In clinic, dose intensification of alkylating agents resulted in increased therapeutic responses, however associated with significant hematological toxicity. Following the development of autologous stem-cells transplantation harvesting from peripheral blood, the high-doses of chemotherapy, initially associated with marked toxic effects, could be more easily tolerated. As a result, the approach was evaluated in different types of solid tumors, including breast, ovarian and germ-cell tumors, small cell lung carcinoma, soft tissue sarcomas and Ewing sarcoma. To date, high-doses chemotherapy with hematopoietic stem-cells support is only used as a salvage therapy to treat poor prognosis germ-cell tumors patients with chemo-sensitive disease. Regarding breast and ovarian cancer, high-doses chemotherapy should be considered only in the context of clinical trials. However, intensive therapy as an approach to overcome resistance to standard treatments is still relevant. Numerous efforts are still ongoing to identify novel therapeutic combinations and active treatments to improve patients' responses.
\end{abstract}

Key words: High-dose chemotherapy; Germ cell tumors; Autologous stem cell transplant; Solid tumors; Breast cancer; Ovarian cancer 


\section{Introduction}

Les bases scientifiques qui ont contribué au développement du concept des hautes-doses de chimiothérapie (HDCT) proviennent d'observations faites initialement in vitro. Dans les années 1980, il a été constaté que l'exposition des cellules tumorales à des doses croissantes de certains médicaments cytotoxiques augmentait leur mort cellulaire. II a été également observé que la résistance acquise des cellules tumorales aux agents akylants pouvait être surmontée par l'intensification des doses. Ce concept a alors été traduit à la clinique et les protocoles de hautesdoses ont été créés pour traiter les tumeurs résistantes ou réfractaires. Par la suite, la compréhension du mécanisme d'action des médicaments alkylants et la possibilité d'administrer des poly-chimiothérapies sont venues renforcer l'utilisation des HDCT [1, 2].

Cela fait désormais plus de vingt ans que les HDCT sont évaluées pour traiter les tumeurs solides (en particulier dans le cancer du sein, de l'ovaire, les tumeurs germinales, le cancer bronchique à petites cellules, les tumeurs cérébrales, les sarcomes des tissus mous et le sarcome d'Ewing), sans que la preuve de son efficacité n'ait été pourtant clairement démontrée. Aujourd'hui, cette approche est utilisée uniquement pour traiter les tumeurs germinales de mauvais pronostique comme thérapie de rattrapage pour les patients dont les tumeurs chimio-sensibles ne répondent pas aux traitements de rattrapage conventionnels.

Le traitement par HDCT expose à une toxicité médullaire sévère, imposant un prélèvement de cellules souches hématopoiétiques puis leur réinjection au cours de cette intensification. Dans les années 1980, grâce aux progrès réalisés en oncohématologie, et en particulier grâce à la possibilité de constituer ex-vivo des réserves de cellules souches hématopoïétiques, un nouveau souffle aux traitements intensifs a été donné. Ainsi, la cancérologie a connu une multiplication de protocoles expérimentaux testant la faisabilité et l'efficacité des chimiothérapies intensives associées à l'autogreffe de cellules souches hématopoïétiques.

Cette procédure simplifiée de prélèvement a en effet considérablement diminué la morbidité et la mortalité liée à l'administration de HDCT, mais a également raccourci le temps d'hospitalisation, réduit les coûts du traitement et a permis l'utilisation de cette pratique en dehors d'essais thérapeutiques. De plus, l'avènement des facteurs de croissance hématopoïétiques a autorisé l'augmentation des doses utilisées des médicaments cytotoxiques.

\section{La résistance à la chimiothérapie}


La résistance aux traitements anti-tumoraux est un problème majeur dans la thérapeutique du cancer. Les tumeurs peuvent en effet présenter une résistance intrinsèque qui survient d'emblée lors de l'administration des premiers cycles de traitement ou développer une résistance, dite alors acquise. Même dans les cas où la thérapie est initialement efficace, les tumeurs développent quasi invariablement une résistance aux médicaments qui s'accompagne de la progression du cancer voire de l'apparition de nouvelles métastases.

La résistance à la chimiothérapie a été observée dès les années 1940 lorsqu'a commencé " l'ère moderne de la chimiothérapie ». A cette époque, Goodman and Gilman [3] ont proposé, pour la première fois, d'utiliser la chimiothérapie pour traiter le cancer. En effet, leurs observations des effets marqués des moutardes azotées sur le tissu lymphoïde couplées avec le fait que les cellules en prolifération étaient sélectivement ciblées par l'action cytotoxique de ces molécules ont incité les chercheurs à utiliser ces composés pour traiter un patient atteint de lymphome. Malgré la diminution des masses tumorales détectables chez ce patient, la rémission n'a duré que quelques semaines avant que la maladie ne progresse de nouveau. Cependant, à partir de ces premières observations, le principe avait été établi et les années suivantes furent consacrées à l'amélioration chimique des agents alkylants (tels que la cyclophosphamide et le chlorambucil) et au développement d'analogues du folate (methotrexate).

Dans les années 1980, Frei et ses collègues [2] ont montré que la résistance à un médicament pouvait être acquise in vitro mais nécessitait néanmoins pour être maintenue le traitement intermittent des cellules tumorales par des faibles concentrations des agents alkylants (nitrosourée et carmustine/BCNU). Lorsque les doses étaient intensifiées (d'environ cinq à dix fois), la résistance pouvait être par contre surmontée. C'est ainsi que l'approche HDCT, basé sur la combinaison de plusieurs agents anticancéreux, a été imaginée puis mise en place avec comme objectif de retarder ou d'empêcher l'émergence de clones résistants [1].

Parmi les mécanismes moléculaires à l'origine de l'échec des thérapies anticancéreuses, les causes pharmacodynamiques liées au transport membranaire des médicaments ont été parmi les plus explorées. Parmi les transporteurs ainsi identifiés, la glycoprotéine-P (P-gp) est certainement l'un des mieux connus et des plus importants d'un point de vue clinique. L'identification de sa surexpression à la surface des cellules tumorales rendues résistantes à divers agents chimiotherapéutiques aux modes d'action pourtant distincts, a permis de définir un phénotype de multirésistance, le MDR (pour multidrug resistance). La P-gp, codée par le gène MDR1/ABCB1 chez l'homme, a en effet un rôle de pompe à efflux, entraînant un flux de médicaments, aux structures chimiques diverses, du cytoplasme vers le milieu extracellulaire [4]. 
La sélection de clones pré-existants de cellules tumorales avec un phénotype MDR, provenant par exemple de réarrangements génomiques dans la région régulatrice du gène MDR1 entraînant sa surexpression, a été pendant longtemps la théorie la plus acceptée pour expliquer l'acquisition d'une résistance. Néanmoins, aujourd'hui, la reconnaissance que les tumeurs présentent également une hétérogénéité phénotypique d'origine non-génétique a permis de mieux comprendre l'apparition précoce et rapide d'un phénotype MDR après chimiothérapie. En effet, ce phénotype de résistance peut être induit indépendamment de toute sélection au sens Darwinien du terme. Dans ce cas, les cellules tumorales peuvent, transitoirement, occuper un stade de résilience au stress cytotoxique et surexprimer MDR1 sans qu'aucune altération génétique du gène MDR1 ne soit nécessaire. Ces modifications épigénétiques qui peuvent alors être transmises lors de mitoses successives aux nouvelles générations de cellules tumorales, pourraient ainsi favoriser l'apparition et l'installation progressive de mutations permettant alors d'inscrire définitivement la résistance dans le génome cellulaire [5]. D'un point de vue clinique, l'installation d'un phénotype transitoire de résistance pourrait contribuer à expliquer un phénomène fréquemment observé dans la thérapie anticancéreuse, la «re-réponse» à un traitement après le développement d'une résistance initiale à ce même traitement [6].

D’autres mécanismes peuvent expliquer également l'échec de la thérapie anticancéreuse systémique. Ainsi, la modification de la structure de la protéine cible du médicament, l'activation de voies alternatives de survie cellulaire ou l'altération des voies de réparation de l'ADN peuvent concourir à une résistance. En particulier, les voies de réparation de l'ADN sont des déterminants majeurs de la réponse tumorale aux agents alkylants. Or, ces voies peuvent être augmentées ou dérégulées aboutissant ainsi à la réduction de la cytotoxicité du médicament par réparation accélérée des lésions. De ce fait, la modulation pharmacologique des mécanismes de réparation constitue une voie, encore peu explorée, pour augmenter l'efficacité thérapeutique de certains médicaments [7].

\section{Cancer du sein}

L'approche HDCT pour traiter le cancer du sein a été élaborée comme schéma thérapeutique pour être appliquée en situation adjuvante ou métastatique. Depuis les années 1970, la prise en charge des patientes atteintes d'un cancer du sein à haut-risque de rechute implique l'administration d'une thérapie adjuvante, avec pour objectif d'éliminer les éventuels résidus tumoraux et de prolonger la survie. Dans cette optique, plusieurs études de phase II ont été conduites dans les années 1980 et le début des années 1990 pour tester l'efficacité des HDCT, complétée par la transplantation de cellules 
souches hématopoïétiques. Le faible nombre de patientes incluses dans chacune des études fait néanmoins que les résultats de ces études restent difficilement extrapolables.

Plus récemment, dans une analyse faite sur l'ensemble des résultats de 15 essais cliniques randomisés réalisés entre 1990 et 2002 et concernant une thérapie adjuvante à base de HDCT, Berry et al [8] ont inclus 6,120 patients [3118 qui ont reçu la HDCT et 3092 qui ont reçu un traitement standard (contrôle)]. L'objectif principal de cette étude était de déterminer si, comme le concluait la plupart des études inclues dans cette analyse, les HDCT n'avaient pas de bénéfices par rapport à la thérapie standard. Pour ce faire, les critères de jugements principaux choisis par les auteurs ont été la SSR (survie sans récidive) et la SG (survie globale). Une réduction du risque de rechute pour le groupe HDCT a été montrée pour 11 des 15 essais analysés. Parmi ces 11 essais, trois ont montré une réduction statistiquement significative en faveur de la HDCT. En ce qui concerne la survie globale, aucune différence statistique entre les deux traitements n'a par contre pu être démontrée, sauf pour un sous-groupe de patientes HER2-negative. Dans ce cas, il y avait une réduction de $21 \%$ du risque de décès. Cette réduction était encore plus marquée $(33 \%)$ chez les patientes triple-négatives (négatives pour les récepteurs aux hormones et pour HER2). Sur un total de 89 décès liés au traitement, 72 (6\%) ont été recensés dans le groupe HDCT et $17(1,4 \%)$ dans le bras contrôle. De plus, dans 6 essais sur les 15 analysés, il a été constaté un total de 33 tumeurs malignes secondaires, classés comme syndrome myélodysplasique ou leucémie myéloïde aigue. Sur les 33 tumeurs, 17 se sont produites dans le groupe HDCT et 16 dans le groupe contrôle. En conclusion, cette étude a montré que la HDCT ne se traduit pas par un bénéfice significatif de la survie globale corroborant les conclusions de la méta-analyse de Farquhar et al publiée en 2007 [9].

En ce qui concerne les situations métastatiques, en France, deux protocoles de phase III ont été lancé en 1995, les programmes PEGASE 03 et 04 (Programme d'Etudes des Greffes Autologues dans les cancers de SEin). Des patientes de moins de 60 ans avec une maladie mesurable et ayant reçu l'épirubicine ou la doxirubicine en thérapie adjuvante ont été incluses. Dans le programme PEGASE 03, les patientes en réponse complète ou partielle ont été randomisées en deux bras après avoir reçu 4 cycles de FEC (A : groupe sans traitement ; B : groupe HDCT par thiotepa, cyclophosphamide). Trois mois après la fin du dernier cycle de chimiothérapie le taux de réponse a été de $59 \%$ et $92 \%(p=0.01)$, respectivement. La survie sans progresssion à 1 an a été de $19 \%$ et $46 \%(p=0.0001)$, respectivement. La survie globale n'a pas été statistiquement significative à 3 ans ( $30 \%$ bras, $38 \%$ bras B). Dans les programme PEGASE 04, les patientes répondeuses ont été randomisées pour recevoir 4 à 6 cycles de chimiothérapie conventionnelle (maintenance) ou HDCT (mitoxantrone, cyclophosphamide, melphalan) avec support de cellules souches hématopoiétiques [10]. Dans cette étude, même si une amélioration de la survie globale a été constatée, la taille réduite de la population incluse sur une 
longue période de temps a empêché de conclure que la HDCT avait un impact significatif sur la survie globale des patientes traitées [11].

De façon similaire à ce qui a été réalisé dans le cadre d'une thérapie adjuvante, l'équipe de Berry [12] a analysé six études randomisées comparant la HDCT avec transplantation de cellules souches à un régime de chimiothérapie à dose conventionnelle sans transplantation dans des situations métastatiques. L'analyse des résultats impliquant les études BBCRG (Berlin Breast Cancer Research Group), ECOG (Eastern Cooperative Oncology Group), IBDIS (International Randomized Breast Cancer Dose Intensity Study), NCIC (National Cancer Institute of Canada) et PEGASE 03/04 a révélé un avantage statistiquement significatif de la HDCT dans la survie sans récidive (médiane 0.91 vs 0.69 ans). L'analyse des sous-groupes a suggéré un bénéfice de la HDCT pour les patientes de moins de 50 ans, pré-ménopausées, avec des métastases des tissus mous, et présentant deux ou plusieurs sites métastatiques. Cependant, les associations entre les covariables étudiées ont été faibles et ont montré une modeste différence dans la survie globale ne permettant pas de conclure à un bénéfice réel de la HDCT.

En conclusion, la plupart des études randomisées, conduite sur des patientes à haut risque ainsi que dans des situations métastatiques, ont rapporté un avantage dans la SSR. Avec l'avènement des thérapies ciblées et des nouvelles modalités de traitement pour le cancer du sein, la publication des résultats d'études randomisées rapportant un bénéfice de la HDCT dans la survie globale est passée presque inaperçue au sein de la communauté médicale.

Toutefois, il est important de noter que certains auteurs estiment qu'un arrêt définitif de la HDCT dans le cancer du sein pourrait être aujourd'hui prématuré si l'on considère que dans le cancer du sein à haut-risque deux grandes études européennes ont montré un bénéfice de la HDCT dans la survie globale de la population HER2-négative [13] et triple-négative [14]. Ainsi, I'approche HDCT dans le traitement du cancer du sein mériterait d'être approfondie chez les patientes HER2-négatives et triple-négatives, dont la maladie est chimio-sensible.

En accord avec cette observation, il a été suggéré récemment, que les bénéfices des HDCT observés dans les groupes de patientes triple-négatives pourraient être associés à un nombre accru, dans ce groupe, de patientes déficientes en protéines impliquées dans la voie de réparation de l'ADN par recombinaison homologue. Les résultats tirés d'une étude rétrospective ont en effet montré que le statut BRCA-like est un facteur prédictif de survie après les HDCT faite à base d'epirubicine, de thiotepa et de cyclophosphamide [15]. Les auteurs suggèrent que le statut BRCA-like pourrait donc être utilisé comme biomarqueur prédictif de réponse aux thérapies HDCT qui emploient les agents alkylants. 


\section{Cancer de l'ovaire}

Les programmes de chimiothérapie à hautes-doses dans le traitement des carcinomes ovariens ont émergé plus tardivement comparés aux études lancées dans le cadre du cancer du sein. En raison de sa chimio-sensibilité importante, mais également d'un taux élevé de rechutes conférant à cette maladie un très mauvais pronostic, le cancer de l'ovaire a été considéré comme une indication possible de l'intensification thérapeutique. Par suite, les intensifications thérapeutiques avec autogreffe de cellules souches se sont développées, l'envahissement médullaire étant exceptionnel au cours de l'évolution de cette maladie.

Pour traiter le cancer de l'ovaire, deux stratégies principales d'utilisation des HDCT ont été développées: en consolidation, après obtention d'une réponse complète ou partielle, ou en rattrapage dans les cas d'une rechute ou dans les formes réfractaires de la maladie.

Les premiers articles publiés sur l'utilisation dans le cancer de l'ovaire de la HDCT adjointe d'une transplantation de cellules souches (isolées à partir de la moelle osseuse ou du sang périphérique) comme thérapie de rattrapage, se sont portés sur des patientes qui présentaient une maladie considérée comme résistante ou réfractaire. Les résultats de ces premières études ont montré que le taux de réponse globale pouvait être considéré comme favorable (50\% à $70 \%)$. La durée de la réponse observée restait néanmoins courte (5 à 10 mois) [16].

A la fin des années 1990, Stiff et al [17] ont rapporté une étude réalisée sur plus de 100 patientes qui présentaient une maladie récurrente. Ces patientes ont été traitées avec différents protocoles de HDCT (70 malades ont reçu une association de carboplatine, mitoxantrone et cyclophosphamide, 25 de melphalan et mitoxantrone et 5 d'autres associations). Les médianes de survies globales observées dans cet essai ont été de 9,6 mois pour les patientes présentant une maladie résistante et de 23,1 mois pour les patientes présentant une maladie sensible aux platines. Cette étude a en outre fortement contribué à l'identification de deux facteurs prédictifs liés à l'utilisation de cette approche. Ainsi, et jusqu'à aujourd'hui, la sensibilité aux platines et la charge tumorale en période pré-greffe sont considérées comme les deux plus importants facteurs prédictifs de survie lorsqu'une thérapie de haute-dose est administrée.

Une maladie résiduelle minimale et une meilleure chimio-sensibilité ont orienté l'utilisation des HDCT en consolidation plutôt que dans le cas des maladies récurrentes. Dans ce cadre, une étude rétrospective [18] a évalué l'intérêt de l'utilisation de la HDCT comme thérapie de consolidation après chirurgie et traitement par les taxanes/platines. Dans cette étude, 103 patientes avec un carcinome de l'ovaire à un stade avancé ont été traitées par la chimiothérapie conventionnelle et 
leur évolution comparée à celle de 60 patientes qui ont reçu la HDCT avec le support de cellules souches hématopoïétiques. Aucun avantage de survie globale des patientes n'a été ici mis en évidence. Néanmoins, la HDCT a été associée à de résultats meilleurs chez les patientes jeunes $(\leq 50$ ans) avec une médiane de survie globale de 54,6 mois contre 36 mois pour les patientes recevant la chimiothérapie conventionnelle. Les résultats prometteurs de ces études pilotes n'ont malheureusement pas été confirmés par les études randomisées de phase III [19].

\section{Tumeurs germinales}

Le traitement de première ligne des tumeurs germinales de mauvais pronostic est une combinaison de bléomycine, d'étoposide et de cisplatine (BEP). Malgré un taux de guérison déjà très élevé avec le protocole BEP, il subsiste encore 10 à $20 \%$ des patients qui rechutent. Ces patients sont alors candidats à un traitement initial de rattrapage tel que le VeIP (vinblastine-ifosfamide-cisplatine), le VIP (une variante du VelP où l'étoposide remplace la vinblastine), ou une combinaison de sels d'ifosfamide et de platine avec le paclitaxel (TIP) avec ou sans chirurgie ultérieure. Une alternative à ces traitements est la thérapie d'intensification.

En effet, la HDCT dans la prise en charge des tumeurs germinales est utilisée depuis les années 1980 mais son succès a été initialement limité par les niveaux élevés de mortalité associée au traitement. Tout comme dans le cas du cancer du sein et de l'ovaire, la collecte des cellules souches à partir du sang périphérique a conduit à d'importantes améliorations d'efficacité du traitement accompagnées par une réduction de la toxicité.

Les études randomisées comparant la HDCT à la chimiothérapie conventionnelle sont néanmoins limitées. A ce jour, le seul essai randomisé qui a inclus 280 patients dans 43 institutions et 11 pays et qui a comparé le remplacement d'un quatrième cycle classique de VIP/VeIP par les HDCT carboplatine-étoposide-cyclophosphamide (CARBOPAC) avec support des cellules souches hématopoiétique n'a montré aucun bénéfice de la SSR ou de la SG [20]. Néanmoins, cette étude a évalué un cycle unique de HDCT. Aujourd'hui, une étude multicentrique randomisée pour tester la supériorité ou non des HDCT en tandem par rapport aux doses standard de chimiothérapie comme première ligne de rattrapage est en cours (ClinicalTrials.gov Identifier NCT02375204). L'étude TIGER souhaite comparer la chimiothérapie standard par TIP versus HDCT en utilisant l'ifosfamide plus paclitaxel comme thérapie de mobilisation suivie par les hautes-doses de carboplatine et etoposide.

En ce qui concerne les facteurs pronostiques de réponse à la $\mathrm{HDCT}$, les patients réfractaires absolus au cisplatine, ceux atteints d'une tumeur germinale non-séminomateuse médiastinale primitive, ceux ayant un taux d'hCG supérieur à $1000 \mathrm{UI} / \mathrm{L}$ ainsi que ceux ayant une maladie progressive ont été 
identifiés comme de mauvais pronostique [21]. Plus récemment, des catégories de risque pour guider le traitement des tumeurs germinales en rechute après une première ligne de chimiothérapie à base de platine ont été établies [22]. Différentes études ont montré que les patients à haut-risque, y compris ceux porteurs de tumeurs non-séminomateuses et présentant une atteinte médiastinale primitive, peuvent être guéris avec la HDCT, ce qui n'est pas le cas avec un traitement par chimiothérapie à dose conventionnelle.

Plusieurs éléments semblent néanmoins déterminants pour améliorer les résultats en termes d'efficacité et de toxicité de la HDCT comme thérapie de rattrapage dans les tumeurs germinales. Le nombre de cycles, le temps d'administration ainsi que les intervalles entre les cycles figurent parmi les variables pronostiques importantes.

Dans cette optique, la faisabilité et l'efficacité de plusieurs cycles de HDCT ont été explorées et établies. Les recommandations de l'European Consensus Conference on diagnosis and treatment of germ-cell cancer [23] préconisent I'utilisation de trois cycles d'un régime HDCT combinant carboplatine et étoposide. Ainsi, le protocole TI-CE (paclitaxel [T] plus ifosfamide [I] suivi par une hautes-dose de carboplatine [C] plus Etoposide $[\mathrm{E}]$ avec support de cellules souches) pour lequel a été rapportée une survie sans récidive à 5 ans de $47 \%$ et une survie globale de $52 \%$ (médiane de suivi de 61 mois) est actuellement considéré comme le régime de choix [24].

Comme alternative de rattrapage pour les patients atteints de tumeurs germinales de mauvais pronostique, les programmes TAXIF $[25,26]$ ont été élaborés. Ces programmes impliquent une étape de mobilisation par doses semi-intensives de la combinasion épirubicine-paclitaxel (EPI-TAX) suivie par trois cycles de HDCT (1 cycle de thiothepa-cyclophosphamide ou thiothepa-paclitaxel et 2 cycles d'ifosfamide-carboplatine-etoposide) administrés aux patients présentant une maladie chimiosensible après EPI-TAX. Par cette approche, il a été montré que la survie sans récidive à deux ans atteignait 50\% [95\% Cl: 32-67] et la survie globale $66 \%$ [95\%Cl: 44-81] [26].

Plus récemment, Nieto et ses collègues [27], afin d'améliorer les résultats pour les patients à haut risque de rechute tardive, ont évalué l'administration de bevacizumab en plus de cycles séquentiels de HDCT. Dans cette étude, 43 patients ont reçu le bevacizumab préalablement à deux cycles HDCT (cycle 1 gemcitabine avec docetaxel-melphalan-carboplatine et cycle 2 ifosfamide-melphalancarboplatine). Par cette approche les auteurs dépassent les résultats précédemment publiés en utilisant les HDCT seules. La survie sans récidive et la survie globale ont atteint $55,8 \%$ et $58,1 \%$, respectivement, avec un suivi médian de 46 mois. Ces résultats ont été obtenus grâce à l'adjonction d'une résection complémentaire de lésions résiduelles dont 9 cas ont montré du tissu tumoral de type tératome ou de tumeur viable. II est à noter que 4 décès liés aux procédures d'intensification ont été observés. 


\section{Conclusion et Perspectives}

Tous les régimes HDCT présentent des toxicités aigues et tardives. Avec l'avènement de la transplantation de cellules souches à partir du sang périphérique en lieu et place des cellules issues de la moelle osseuse, la tolérance à la thérapie intensive a été énormément améliorée et divers protocoles de HDCT pour traiter les tumeurs solides ont été créés.

Actuellement, pour ce qui concerne les tumeurs solides, la HDCT n'est considérée comme option thérapeutique que dans le cas du traitement des tumeurs germinales de mauvais pronostique. Elle y est utilisée comme une thérapie de rattrapage après 1 ou 2 lignes de chimiothérapie donnée à des doses conventionnelles. Les tumeurs germinales font en effet partie de tumeurs solides parmi les plus chimio-sensibles et qui présentent la singularité de pouvoir guérir avec la chimiothérapie à hautes-doses. Cependant, il est important de noter que l'administration de ce type de thérapie intensive, non dépourvue d'effets toxiques, est réservée aux centres experts, assurant une prise en charge optimale des patients.

En dehors des tumeurs germinales, diverses autres indications ont été testées. Dans le cancer du sein, la plupart des études utilisant les thérapies de hautes-doses ont montré une amélioration de la survie sans récidive, la survie globale restant quant à elle inchangée. Néanmoins, aujourd'hui, l'utilisation de la HDCT pour traiter le cancer du sein a diminué de manière significative. De façon similaire, dans le cadre du traitement du cancer de l'ovaire, même si parfois un taux de réponses élevé a pu être observé, les réponses restent généralement brèves et les survies prolongées sans rechute rares. L'absence de bénéfice dans la survie globale fait conclure que la thérapie intensive n'a pas d'indication claire pour ces types de tumeurs en dehors de la mise en place de nouveaux essais thérapeutiques. Ainsi, pour ces indications, d'autres stratégies thérapeutiques sont actuellement en cours d'évaluation pour contourner la résistance aux traitements standard. Dans le cancer de l'ovaire, la thérapie métronomique par administration de chimiothérapie associée au bévacizumab, l'ajout de l'immunothérapie et l'utilisation des inhibiteurs de PARP sont des exemples.

Les résultats de la thérapie HDCT peuvent évoluer dans les prochaines années, notamment si de nouveaux médicaments sont introduits dans les régimes HDCT. Des molécules telles que les antiangiogéniques, les inhibiteurs PARP, voire l'immunothérapie pourraient en effet jouer un rôle décisif dans l'élaboration et la réalisation de nouveaux essais thérapeutiques impliquant la HDCT.

Dans cette idée, a été récemment lancé un essai clinique combinant HDCT et molécules ciblées antiangiogéniques pour traiter les tumeurs germinales (ClinicalTrials.gov Identifier NCT01966913). Le VEGF est en effet surexprimé dans ce type de pathologie et représente un facteur de risque 
indépendant chez ces patients. Des dosages sériques du VEGF ont montré que ce marqueur est significativement plus élevé chez les patients porteurs de tumeurs germinales en comparaison au groupe contrôle mais aussi qu'il existe une augmentation significative chez les patients porteurs d'une tumeur germinale métastatique en comparaison aux patients porteurs d'une maladie non métastatique [28]. En outre, il est connu que l'administration de bevacizumab avec des doses conventionnelles de chimiothérapie est synergique, au moins en partie, car le bevacizumab réduit la pression du liquide interstitiel intratumoral et améliore la délivrance des molécules cytotoxiques au sein de la tumeur [29]. Ainsi, son administration avec les HDCT devrait, en augmentant la concentration intracellulaire des médicaments, optimiser leurs effets.

De même, pour ce qui concerne les molécules ciblant les voies de réparation de l'ADN, les inhibiteurs PARP semblent être une voie prometteuse à inclure dans le design de nouveaux protocoles cliniques impliquant les doses intensives de chimiothérapie.

En effet, des expériences de létalité synthétique ont déjà fourni la preuve de l'utilité de ces molécules dans le contexte de tumeurs présentant un déficit de réparation de l'ADN par recombinaison homologue $(\mathrm{RH})$. Or, sachant qu'environ $15 \%$ des tumeurs ovariennes présentent des déficiences dans ce mécanisme de réparation en raison, notamment, de mutations inactivatrices des gènes BRCA, le ciblage sélectif des cellules tumorales par les inhibiteurs PARP devrait conduire à l'accumulation de cassures double brins de I'ADN dans les cellules BRCA déficientes et s'accompagner d'un effet cytotoxique prononcé.

Les récentes données du projet Cancer Genome Atlas renforcent également ce nouveau rationnel thérapeutique. Les travaux publiés par ce consortium suggèrent qu'au-delà des mutations germinales, les cancers de l'ovaire séreux de haut-grade présentent aussi des altérations fonctionnelles de la voie de $\mathrm{RH}$ qui surviennent par soit des mutations somatiques affectant les gènes BRCA1/2, soit des modifications épigénétiques, ou par le dysfonctionnement d'autres protéines impliquées dans cette voie. Ainsi, alors que seule une petite proportion (10-15\%) de patientes est porteuse des mutations germinales BRCA1/2, près de la moitié de l'ensemble des cancers de l'ovaire séreux de haut-grade présenterait en réalité des défauts dans la voie de RH. Cette observation implique donc que les inhibiteurs de PARP pourraient avoir une utilité clinique dans un groupe de patientes atteintes de cancer de l'ovaire bien plus vaste qu'initialement imaginé.

Aujourd'hui, l'identification du phénotype de déficience en RH est possible grâce à la détermination du statut BRCA-like ou BRCAness des tumeurs, et ce, même en absence de mutations germinales BRCA1/2. Ce progrès a été rendu possible grâce au développement de techniques de génomique à haut débit telles que le CGH (comparative genomic hybridization) ou le SNP (single-nucleotide polymorphism genotyping and deep sequencing). 
Dans le cancer du sein, par exemple, la détermination du profil BRCA1-like a permis d'identifier les patientes les plus susceptibles de bénéficier d'une thérapie à hautes-doses faite à base d'agents alkylants. Chez les patientes atteintes d'un cancer du sein de stade III et qui présentent un profil BRCA1-like, la SSR à 7-ans est ainsi passée de 30\% à 78\% lorsque les patientes ont été traitées par les HDCT (adjusted hazard rate (HR) 0.12, p=0.001), tandis que, pour les patientes non-BRCA1-like, aucun bénéfice n'a pu être observé [15]. En raison des données récemment publiées indiquant que les inhibiteurs PARP sont actifs chez les patientes atteintes d'un cancer du sein BRCA1-like, il est probable que ces molécules aient un effet synergique une fois combinées avec les HDCT faites à base d'agents alkylants.

L'immunothérapie, par le blocage de l'axe PD-1/PD-L1, pourrait également être une option dans le traitement des tumeurs primaires du testicule. Les patients avec un mauvais pronostique et qui sont aujourd'hui orientés vers une thérapie de rattrapage à hautes-doses pourraient bénéficier des inhibiteurs des points de contrôles immunologiques. En effet, tout dernièrement, il a été montré que l'expression de PD-L1 est augmentée chez les patients qui présentent une maladie à un stade avancé et des caractéristiques de mauvais pronostique. Ces résultats suggèrent une tolérance immunitaire pouvant faciliter la progression tumorale médiée par l'expression accrue de PD-L1 [30].

Enfin, l'identification de marqueurs tumoraux propres aux caractéristiques moléculaires des tumeurs de chaque patient pourraient constituer une approche innovante pour le développement et I'utilisation future des HDCT. L'utilisation de biopsies liquides pourrait, par exemple, permettre de monitorer, en temps réel, les réponses au traitement à l'image de ce qui a été récemment montré pour les cancers colorectaux et du poumon. Ainsi, une biopsie liquide réalisée au début du traitement puis de manière régulière en cours et après le traitement pourrait permettre $d$ 'anticiper la progression et la rechute, et ce, avant que les signes cliniques ou radiologiques n'apparaissent, offrant la possibilité de modifier le traitement précocement. 


\section{Références}

1. DeVita VT, Schein PS. The use of drugs in combination for the treatment of cancer: rationale and results. N Engl J Med. 1973; 288(19):998-1006.

2. Frei E 3rd, Cucchi CA, Rosowsky A, Tantravahi R, Bernal S, Ervin TJ, Ruprecht RM, Haseltine WA. Alkylating agent resistance: in vitro studies with human cell lines. Proc Natl Acad Sci U S A. $1985 ; 82(7): 2158-62$.

3. Gilman A. The initial clinical trial of nitrogen mustard. Am J Surg. 1963; 105:574-8.

4. Gillet, J. P. \& Gottesman, M. M. Mechanisms of multidrug resistance in cancer. Methods Mol. Biol. 2010; 596, 47-76.

5. Pisco AO, Brock A, Zhou J, Moor A, Mojtahedi M, Jackson D, Huang S. NonDarwinian dynamics in therapy-induced cancer drug resistance. Nat Commun. 2013; 4:2467.

6. Kuczynski EA, Sargent DJ, Grothey A, Kerbel RS. Drug rechallenge and treatment beyond progression--implications for drug resistance. Nat Rev Clin Oncol. 2013 Oct;10(10):57187.

7. Helleday T, Petermann E, Lundin C, Hodgson B, Sharma RA. DNA repair pathways as targets for cancer therapy. Nat Rev Cancer. 2008; 8(3):193-204.

8. Berry DA, Ueno NT, Johnson MM, Lei X, Caputo J, Rodenhuis S, Peters WP, Leonard RC, Barlow WE, Tallman MS, Bergh J, Nitz UA, Gianni AM, Basser RL,Zander AR, Coombes RC, Roché H, Tokuda Y, de Vries EG, Hortobagyi GN, Crown JP, Pedrazzoli P, Bregni $\mathrm{M}$, Demirer T. High-dose chemotherapy with autologous stem-cell support as adjuvant therapy in breast cancer: overview of 15 randomized trials. J Clin Oncol. 2011; 29(24):321423.

9. Farquhar CM, Marjoribanks J, Lethaby A, Basser R. High dose chemotherapy for poor prognosis breast cancer: systematic review and meta-analysis. Cancer Treat Rev. 2007; 33(4):325-37.

10. Roche H, Viens P, Biron P, Lotz JP, Asselain B; PEGASE Group. High-dose chemotherapy for breast cancer: the French PEGASE experience. Cancer Control. 2003 JanFeb;10(1):42-7.

11. Lotz JP, Curé $H$, Janvier $M$, Asselain B, Morvan $F$, Legros $M$, Audhuy $B$, Biron $P$, Guillemot M, Goubet J, Laadem A, Cailliot C, Maignan CL, Delozier T, Glaisner S, Maraninchi D, Roché $\mathrm{H}$, Gisselbrecht $\mathrm{C}$. High-dose chemotherapy with haematopoietic stem cell transplantation for metastatic breast cancer patients: final results of the French multicentric randomised CMA/PEGASE 04 protocol. Eur J Cancer. 2005; 41(1):71-80.

12. Berry DA, Ueno NT, Johnson MM, Lei $X$, Caputo J, Smith DA, Yancey LJ, Crump M, Stadtmauer EA, Biron P, Crown JP, Schmid P, Lotz JP, Rosti G, Bregni M, Demirer T. High-dose chemotherapy with autologous hematopoietic stem-cell transplantation in metastatic breast cancer: overview of six randomized trials. J Clin Oncol. 2011; 29(24):3224-31.

13. Rodenhuis $S$, Bontenbal $M$, van Hoesel QG, Smit WM, Nooij MA, Voest EE, van der Wall E, Hupperets $P$, van Tinteren H, Peterse JL, van de Vijver MJ, de Vries EG. Efficacy of highdose alkylating chemotherapy in HER2/neu-negative breast cancer. Ann Oncol. 2006; 17(4):588-96.

14. Gluz O, Nitz UA, Harbeck N, Ting E, Kates R, Herr A, Lindemann W, Jackisch C, Berdel WE, Kirchner H, Metzner B, Werner F, Schütt G, Frick M, Poremba C,Diallo-Danebrock R. Triple-negative high-risk breast cancer derives particular benefit from dose intensification of adjuvant chemotherapy: results of WSG AM-01 trial. Ann Oncol. 2008; 19(5):861-70.

15. Schouten PC, Gluz O, Harbeck N, Mohrmann S, Diallo-Danebrock R, Pelz E, Kruizinga J, Velds A, Nieuwland M, Kerkhoven RM, Liedtke C,Frick M, Kates R, Linn SC, Nitz U, Marme F. BRCA1like profile predicts benefit of tandem high dose epirubicin-cyclophospamide-thiotepa in high risk breast cancer patients randomized in the WSG-AM01 trial. Int J Cancer. 2016 Mar 4. [Epub ahead of print] 
16. Makatsoris T, Seiden MV. High-Dose Therapy for Ovarian Carcinoma. Oncologist. 1997; 2(5):330-339.

17. Stiff PJ, Bayer R, Kerger C, Potkul RK, Malhotra D, Peace DJ, Smith D, Fisher SG. High-dose chemotherapy with autologous transplantation for persistent/relapsed ovarian cancer: a multivariate analysis of survival for 100 consecutively treated patients. J Clin Oncol. 1997; 15(4):1309-17.

18. Sabatier R, Gonçalves A, Bertucci F, Capiello MA, Rousseau F, Lambaudie E, Chabannon $C$, Viens $P$, Extra JM. Are there candidates for high-dose chemotherapy in ovarian carcinoma? J Exp Clin Cancer Res. 2012; 31:87.

19. Möbus V, Wandt H, Frickhofen N, Bengala C, Champion K, Kimmig R, Ostermann H, Hinke A, Ledermann JA; AGO-Ovar/AIO; EBMT. Phase III trial of high-dose sequential chemotherapy with peripheral blood stem cell support compared with standard dose chemotherapy for first-line treatment of advanced ovarian cancer: intergroup trial of the AGO-Ovar/AIO and EBMT. J Clin Oncol. 2007; 25(27):4187-93.

20. Pico JL, Rosti G, Kramar A, Wandt H, Koza V, Salvioni R, Theodore C, Lelli G, Siegert W, Horwich A, Marangolo M, Linkesch W, Pizzocaro G, Schmoll HJ,Bouzy J, Droz JP, Biron P. A randomised trial of high-dose chemotherapy in the salvage treatment of patients failing firstline platinum chemotherapy for advanced germ cell tumours. Ann Oncol. 2005; 16(7):1152-9.

21. Beyer J, Kramar A, Mandanas R, Linkesch W, Greinix A, Droz JP, Pico JL, Diehl A, Bokemeyer C, Schmoll $\mathrm{HJ}$, Nichols CR, Einhorn LH, Siegert W. High-dose chemotherapy as salvage treatment in germ cell tumors: a multivariate analysis of prognostic variables. J Clin Oncol. 1996 Oct;14(10):2638-45.

22. Lorch A, Beyer J, Bascoul-Mollevi C, Kramar A, Einhorn LH, Necchi A, Massard C, De Giorgi U, Fléchon A, Margolin KA, Lotz JP, Germa Lluch JR, Powles T, Kollmannsberger CK. Prognostic factors in patients with metastatic germ cell tumors who experienced treatment failure with cisplatin-based first-line chemotherapy. J Clin Oncol. 2010; 28(33):4906-11.

23. Beyer J, Albers P, Altena R, Aparicio J, Bokemeyer C, Busch J et al. Maintaining success, reducing treatment burden, focusing on survivorship: highlights from the third European consensus conference on diagnosis and treatment of germ-cell cancer. Ann Oncol. 2013; 24(4):878-88.

24. Feldman DR, Sheinfeld J, Bajorin DF, Fischer P, Turkula S, Ishill $N$ et al. TI-CE high-dose chemotherapy for patients with previously treated germ cell tumors: results and prognostic factor analysis. J Clin Oncol. 2010; 28(10):1706-13.

25. Lotz JP, Bui B, Gomez F, Théodore C, Caty A, Fizazi K, Gravis G, Delva R, Peny J, Viens P, Duclos B, De Revel T, Curé H, Gligorov J, Guillemaut S, Ségura C, Provent S, Droz JP, Culine $S$, Biron P. Sequential high-dose chemotherapy protocol for relapsed poor prognosis germ cell tumors combining two mobilization and cytoreductive treatments followed by three high-dose chemotherapy regimens supported by autologous stem cell transplantation. Results of the phase II multicentric TAXIF trial. Ann Oncol. 2005; 16(3):411-8.

26. Selle F, Wittnebel S, Biron P, Gravis G, Roubaud G, Bui BN, Delva R, Bay JO, Fléchon A, Geoffrois L, Caty A, Soares DG, de Revel T, Fizazi K, Gligorov J, Micléa JM, Dubot C, Provent S, Temby I, Gaulet M, Horn E, Brindel I, Lotz JP. A phase II trial of high-dose chemotherapy (HDCT) supported by hematopoietic stem-cell transplantation (HSCT) in germ-cell tumors (GCTs) patients failing cisplatin-based chemotherapy: the Multicentric TAXIF II study. Ann Oncol. 2014; 25(9):1775-82.

27. Nieto $Y$, Tu SM, Bassett R, Jones RB, Gulbis AM, Tannir N, Kingham A, Ledesma C, Margolin K, Holmberg L, Champlin R, Pagliaro L. Bevacizumab/high-dose chemotherapy with autologous stem-cell transplant for poor-risk relapsed or refractory germ-cell tumors. Ann Oncol. 2015; 26(10):2125-32.

28. Bentas W, Beecken WD, Glienke W, Binder J, Schuldes H. Serum levels of basic fibroblast growth factor reflect disseminated disease in patients with testicular germ cell tumors. Urol Res. 2003; 30(6):390-3. 
29. Jain RK. Normalizing tumor vasculature with anti-angiogenic therapy: a new paradigm for combination therapy. Nat Med. 2001; 7(9):987-9.

30. Cierna Z, Mego M, Miskovska V, Machalekova K, Chovanec M, Svetlovska D, Hainova K, Rejlekova K, Macak D, Spanik S, Ondrus D, Kajo K,Mardiak J, Babal P. Prognostic value of programmed-death-1 receptor (PD-1) and its ligand 1 (PD-L1) in testicular germ cell tumors. Ann Oncol. 2016; 27(2):300-5. 